bony cavity cleaned. The graft is then inserted. The edges of the skin incisions are then freshened and sutured.

(5) To stop hæmorrhage from an injured lateral sinus. The graft, here, is used instead of the usual iodoform plug.

\title{
CASE OF HYDROCELE OF FEMORAL HERNIAL SAC.
}

WOMAN, aged 47 .

BY T. MEYRICK THOMAS, M.CH., F.R.c.s.

Two years' history of lump, right groin, getting gradually larger and recently painful.

Past History.-Previous operation for cure of right inguinal hernia.

Examination showed an oval, soft, fluctuating, translucent, irreducible swelling, $\mathrm{I}_{2} \frac{1}{2}$ in. by $\mathrm{I}$ in., in the right groin, lying for the most part below Poupart's ligament, but overlying it to a slight extent and to the outer side of the pubic spine.

There was a slight impulse on coughing.

Most of the candidates diagnosed femoral hernia, but considered it to be an irreducible epiplocele, whereas the soft cystic feel and translucency should have indicated the true nature of the condition.

One or two candidates, recognizing the fluid consistency of the swelling, made the diagnosis of cyst of the canal of Nuck. This cundition should have been excluded by the situation of the swelling, particularly its relation to the pubic spine. The treatment advocated was in practically every instance correct-radical cure by the "high" operation (via the inguinal route), and suturing conjoined tendon to Astley Cooper's ligament.

This operation was carried out a few days after the demonstration and the diagnosis confirmed.

\section{COMMENTS ON A CASE OF MULTIPLE CHRONIC INFLAMMATORY TUMOURS.}

BY HERMON TAYLOR, M.CH., F.R.c.s.

THIS case which I showed at the Fellowship of Medicine provided a good exercise in History. examination procedure from the candidate's point of view. The case was that of a man, aged 54, who had his first illness in 1927 , which consisted of an attack of painless hæmaturia. Following this, he had hæmaturia occasionally until June, 1930, when he was investigated in St. Thomas's Hospital. He was found to have multiple papillomata of the bladder. These were removed by suprapubic cystostomy.

He was subsequently followed-up for four months, after which he was lost sight of until November, I93I, when a further bout of severe hæmaturia brought him back to hospital, and a second suprapubic cystostomy revealed a totally inoperable and extensive carcinoma of the bladder.

While convalescing from the operation, he had a severe chest complication, which confined him to bed in hospital for fourteen weeks, after which time he was discharged and put on the waiting list for deep X-ray therapy.

Five months ago, in January, 1932, while he was still convalescing, he noticed swellings gradually increasing in size on the right knee and left elbow.

Five weeks ago another swelling developed on his right shin, and he began to get pain in the right hip. He was, however, much stronger than he had been, and had put on $3 \frac{1}{2}$ st. in weight since his last operation.

I first saw him on account of the swellings, which were getting bigger. 
An examination of the right knee showed an ill-defined swelling, firm, and fixed to the bone on the inner condvle of the tibia. It was tender only on

Clinical Examination. firm pressure and did not fluctuate. The nodule on the tibia could not be examined as I had explored it two days previously, but it showed the same characters.

The left elbow was disorganized and there was considerable effusion in the joint, so much so that pressure on one side caused the opposite side of the elbow to bulge, and on careful examination "melon-seed" bodies could be felt to slip under the fingers. The periarticular tissues were thickened and nedematous, and the skin was slightly warmer than that over the opposite elbow.

A fourth tumour was discovered, of which the patient had not been aware, which was well defined, firm and elastic, not tender, did not fluctuate, and was attached to the fascia below the right scapula.

Examination of the hip showed no gross deformity, but some pain on full extension, abduction or external rotation of the joint.

The interest of this case lies in the discussion of the diagnosis, particularly from an examination candidate's point of view. In an obviously difficult problem of this sort, the examiner does not expect the candidate to give an opinion at once. What he does expect is a careful clinical examination and a reasoned consideration of the evidence before a diagnosis is suggested.

The majority of the men made a rather poor clinical examination, particularly of the elbow-joint, and arrived too hastily at a diagnosis of secondary carcinomatosis, without sufficient criticism of this diagnosis in view of the facts of the case.

Although one hesitates to diagnose two diseases in one patient, so that one tends to favour a diagnosis of carcinomatous metastases, there are the following points of contra-indication to be considered. Firstly, the patient was not ill enough, having puto on $3 \frac{1}{2}$ st. in weight since he left hospital. Secondly, the swellings varied in sizes somewhat from time to time, and thirdly, the type of carcinoma of the bladder was noto one which gives rise to metastases.

Carcinoma of the bladder can be divided into three pathological types, namely, the sessile nodule, the infiltrating ulcerative type, and the malignant papilloma. Only the first two of these ever give rise to distant metastases, although when they do so they show a preference for the bony skeleton. The malignant papillomata do not give rise to secondary deposits except by implantation into another part of the urinary tract or into an operation wound.

This patient had quite definitely a malignant papilloma type of growth, so that this again was a point of evidence against secondary carcinoma.

Investigation. Investigation of the case revealed further evidence against a diagnosis of secondary carcinoma.

(a) X-ray.-Radiograms of the elbow showed subluxation of the joint with diminished joint space. Some calcification of the synovial villi could be seen and periostitis in the lower end of the humerus and the upper ends of the radius and ulna.

$\mathrm{X}$-ray of the knee showed slight periosteal thickening in the region of the swelling on the tibial condyle and X-ray of the hip showed periosteal thickening along the upper border of the acetabulum, while X-ray of the nodule on the shaft of the right tibia showed a small irregular cavity in the corticalis of the bone, surrounded by a zone of sclerosis and with a track leading from the cavity to the overlying periosteum, which was thickened. This appearance could not have been due to a secondary deposit, which is typically a clear-cut, regular, rounded erosion of the bone, with no surrounding bony reaction. The appearance of this cavity is essentially that due to a chronic pyogenic infection.

(b) The Wassermann reaction was negative.

(c) Aspiration of the elbow-joint withdrew a turbid fluid which contained polymorphonuclear leucocytes, but was sterile on culture.

(d) I had explored the swelling on the tibia two days previously and had found a small bunch of granulation tissue pouting out of the cavity in the corticalis of the tibia 
and lying underneath the thickened œedematous periosteum. Culture of this granulation tissue produced a growth of hæmolytic Staphylococcus aureus.

Cystoscopy showed the bladder to be full of masses of growth involving the greater part of the wall of the bladder.

The key to the problem was the patient's long post-operative illness, he having had a blood-stream infection from the cystitis associated with the extensive ulceration of his bladder, which had given rise firstly to his post-operative pneumonia and secondly to these multiple nodules, which were all inflammatory in nature-manifested typically in the X-ray appearance of the cavity in the corticalis of the tibia.

The organism was apparently the Staphylococcus aureus, and it is typical of mild chronic infections by this organism that they tend to infect the periosteum and synovial membranes, producing multiple inflammatory lesions of the type exemplified in this patient.

The criticism I have to make of the way this case was tackled by the men is that they were too ready to make the facts agree with their preconceived tempting idea of secondary carcinomatosis, neglecting to consider the general health of the patient, the type of growth of the bladder, the unusual combination of sites for the metastases, and the X-ray appearances of the cavity in the corticalis of the tibia.

The swelling under the right scapula was agreed by everyone to be a fibrolipoma.

In view of the hopeless nature of the case, the only line of treatment is an autogenous vaccine prepared from the culture from the granulation tissue.

\section{General Post-Graduate News.}

IT should be noted that Courses arranged by the Fellowship of Medicine are open only to Members unless otherwise staied. A copy of each detailed syllabus is sent to every Member.

To ensure admission or to avoid cancellation of the Courses applications must be made by the date given on each syllabus.

Medicine, Surgery and the Specialities: July 4 to 16.-Prince of Wales's General Hospital Group. All day. Fee $£ 5$ ss. or $£ 33$ s. for one week only.

Proctology : July 4 to 9.-St. Mark's Hospilal. All day. Fee $£ 33 \mathrm{~s}$.

Ophthalmology: July 6 and 7.-Royal Westminster Ophthalmic Hospital. Demonstration on Wednesday, July 6, at 5 p.m., by Mr. G. G. Penman on "Fundi of Medical Interest" ; on Thursday, July 7, at 8.30 p.m., by Mr. C. L. Gimblett on "Some Points in Medical Ophthalmology" (illustrated by epidiascope). Fee 10s. 6d. per demonstration. (Specially suitable for M.R.C.P. candidates.)

Migraine : July 14, 21, 28 (Thursdays)._Medical Society of London at 5 p.m. Lectures for General Practitioners by Colonel R. H. Elliot, M.D. Free to Members of the Fellowship of Medicine. Fee to Non-Members 10s. 6d. the series, or 5s. per lecture.

Indigestion : July 22 at the National Temperance Hospital, at 8.30 p.m.-DiscussionDemonstration for General Practitioners on "Certain Aspects of Indigestion," by a Surgeon, Physician, Radiologist and Pathologist. Free to Members of the Fellowship of Medicine. Fee to Non-Members 5s.

The following doctors have recently arrived from New Zealand: Dr. Elizabeth Bryson (Wellington); Dr. M. S. Wells (Christchurch); Dr. H. Stewart Douglas (Hamilton); Dr. E. M. Litchfield (Wellington); Dr. W. H. Simpson (Wellington).

The following doctors have recently arrived from. Canada: Dr. H. H. Hepburn (Edmonton) ; Dr. G. D. Porter (Toronto) ; Dr. Percy Lowe (Kingston) ; Dr. J. W. Gilchrist (London); Dr. A. L. Anderson (Saskatoon); Dr. Frank J. Day (Toronto); Dr. Norman W. Wrong (Toronto); Dr. A, Gould (Montreal). 